\title{
Integrated Correlative Light Electron Microscopy for Automated Array Tomography
}

\author{
R. Lane ${ }^{1}$, P. de Boer ${ }^{2}$, B. N. G. Giepmans ${ }^{2}$, J.P. Hoogenboom ${ }^{1}$ \\ ${ }^{1}$ Imaging Physics, Delft University of Technology, Delft, the Netherlands \\ ${ }^{2}$ Cell Biology, University Medical Centre Groningen, Groningen, the Netherlands
}

The past several years have seen vast improvements in scale of three-dimensional imaging of biological structures with electron microscopy. As three-dimensional imaging of cell structures continues to advance, correlative light electron microscopy (CLEM) is often looked to for localizing protein or molecular-specific information within the cellular ultrastructure. While the scale of CLEM techniques continues to increase, difficulties such as region-of-interest tracking, resolution mismatch, overlay accuracy, and intermediate sample preparation often arise when using separate imaging systems [1]. To combat these challenges, we have taken the approach of using a high numerical aperture fluorescence microscope integrated inside a scanning electron microscope for array tomography [2]. Using our integrated system, we are developing an automated procedure for acquiring and registering a series of light and electron images matched in axial resolution by the section thickness, allowing for a scalable approach to large-scale correlative imaging.

The integrated system allows for the simultaneous observation of a region of interest on a sample with both FM and EM. The optical axes of both imaging systems can be mechanically aligned to within one micron of one another. An automated registration procedure using cathodoluminescence pointers is used to further optimize the FM-EM correlation to less than 10nm [3]. The correlative imaging pipeline using the integrated system consists of two primary steps. First, sequential FM and EM images are taken at comparable field widths $(\sim 150 \mathrm{~nm} / \mathrm{px})$ in a grid-like fashion. The fluorescent image is taken first to avoid electronbleaching. The translation stage upon which the sample is mounted is moved between images while the microscopes remain stationary. Next, sequential EM images are taken in a second, more refined grid at much higher resolution $(\sim 5 \mathrm{~nm} / \mathrm{px})$. Both the large-scale FM and high resolution EM images share a small overlap with their neighbouring images so that a composite image can be stitched together using pairwise phase correlation. The translation matrices found using phase correlation are then optimized by computing the normalized cross-correlation between image pairs [4]. The high resolution EM images are then registered to the larger field of view EM images so that the resulting composite image is a highly precise correlative map between imaging modalities.

Pancreatic tissue sections from diabetes-prone rats are used to test the applicability of our approach for biomedical research purposes. As the LED light source of the fluorescent microscope supports multiple excitation wavelengths, the $80 \mathrm{~nm}$ tissue section is immunolabelled with multiple labels following resin embedding. Labels include Hoechst for cell nuclei, Alexa488 for amylase, and Alexa594 for insulin, in addition to osmium tetroxide staining for EM. 80nm thick sections are cut and mounted on ITO-coated glass for use in the integrated microscope.

Currently our correlative workflow is operational at small scales in two dimensions. By incorporating serial section array tomography into our method we will be able to extend the process to produce three-dimensional, high resolution, correlative reconstructions. 
References:

[1] LM Collinson, EC Carroll, and JP Hoogenboom, Current Opinion in Biomedical Engineering 3 (2017), p. 49

[2] AC Zonnevylle et al, Journal of Microscopy 252 (2013), p. 58

[3] MT Haring et al, Scientific Reports 7 (2017), p. 43621.

[4] S Preibisch, S Saalfeld, and P Tomancak, Bioinformatics 25 (2009), p. 1463.

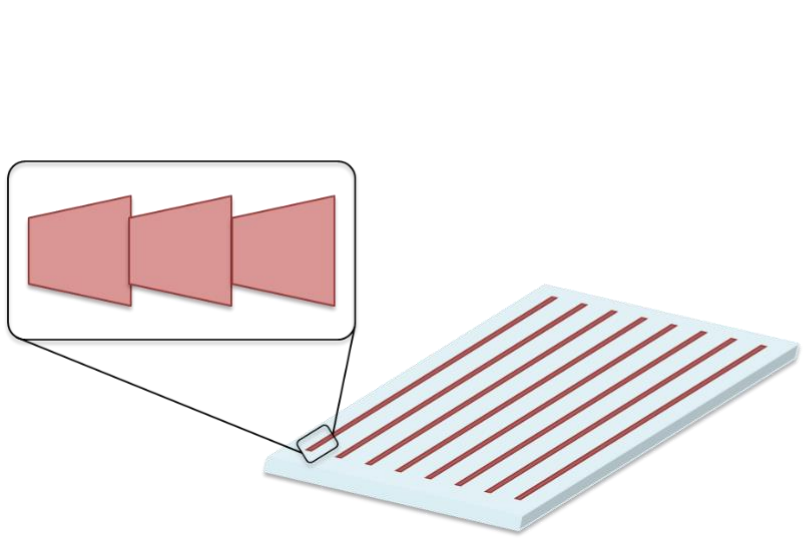

(a)

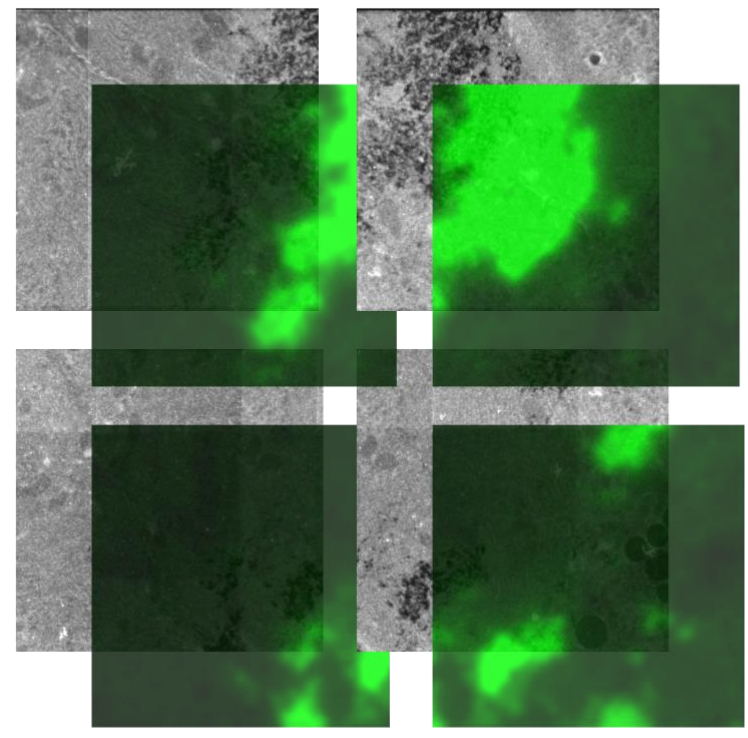

(c)

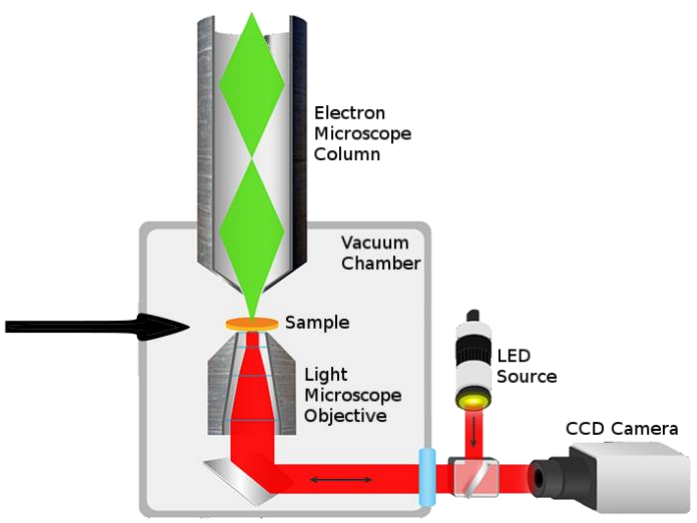

(b)

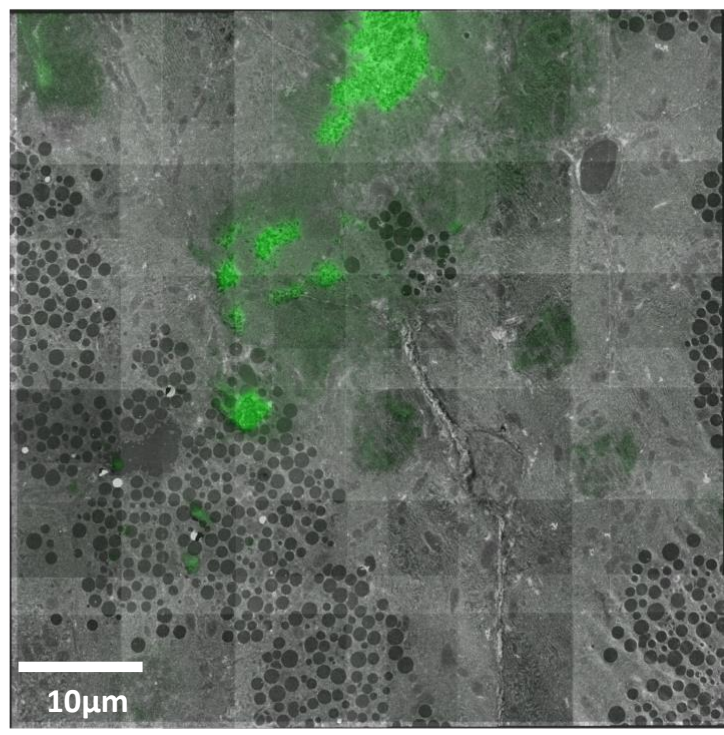

(d)

Figure 1. (a) Serial sections of specimen are collected as ribbons and mounted on ITO-coated slide. (b) ITO-coated slide inserted into vacuum chamber of integrated microscope. (c) Sequential FM and EM images are taken automatically as stage is translated following automated alignment. (d) FM and EM images are stitched together to form composite, correlative image. 